\title{
Diagnosis and management of Ogilvie's Syndrome post caesarean section: case report
}

\author{
Dr Leah Hawkins, MBBCh, Foundation Year 2, FGH \\ Mr Sunny Ajayi, DFSRH, PGCE (Med Ed), MRCOG, Consultant Obstetrician \& Gynaecologist, FGH
}

\begin{abstract}
A 36-year-old woman presented to the maternity unit two days post caesarean section (CS) with abdominal distension, pain and constipation. She was found to be septic on admission. Imaging demonstrated dilated bowel loops without an identifiable site of obstruction highlighting Ogilvie's syndrome (OS) as the cause of her symptoms. Hospital acquired pneumonia (HAP) was identified as the source of infection with accompanying right sided lower lobe collapse. She was reviewed by multiple specialties to aid management and was subsequently managed conservatively for pseudo-obstruction. She made a good recovery and was able to return home after 10 days in hospital.
\end{abstract}

\section{BACKGROUND}

OS, also known as acute colonic pseudo-obstruction (ACPO), is characterised by massive dilatation of the colon without an obvious point of obstruction. ${ }^{1,2}$ Symptoms are similar to those demonstrated with colonic obstruction, which include abdominal pain, distension, nausea, vomiting and constipation. It has been suggested that it is a known complication following surgery, usually following obstetric/gynaecological and orthopaedic surgery but can also occur with infection or following cardiac events. ${ }^{1}$ $10 \%$ of patients diagnosed with ACPO have undergone obstetric/gynaecological procedures..$^{2,3}$

OS is a rare complication of obstetric surgery - in this case following CS. A multidisciplinary team approach was integral, and the teams involved throughout the patient's stay included obstetrics, general surgery, general medicine, microbiology, physiotherapy, dietetics and radiology.

This is an important case to highlight, not only because of the rarity of the condition, but also because of the potentially fatal complications that can arise from it in terms of perforation, ischaemia and mortality. Also specific to this case were the hurdles faced which arose from the coronavirus pandemic at the time.

\section{CASE PRESENTATION}

A 36-year-old woman presented to the assessment unit two days post CS after being discharged the previous day. She had one previous normal vaginal birth and two CS. On review she was found to have severe central abdominal pain and had not opened her bowels or passed flatus since before the operation. On examination, she looked unwell, abdominal examination revealed a grossly distended abdomen, and no bowel sounds were heard on auscultation. She was found to have rebound tenderness of the abdomen but her CS scar was found to be clean. She reported no PV loss or bleeding and there was no nausea or vomiting.

Initial observations triggered for sepsis; most notably she was tachycardic at $120 \mathrm{bpm}$ and hypoxic at $93 \%$ but afebrile at presentation. Chest auscultation revealed decreased air entry at the bases but no discernible crackles. She had no obvious initial symptoms pointing to the site of infection but the appropriate work up was performed accordingly.

Her stay was further complicated as she was screened for COVID-19. Whilst awaiting the results of the swabs, she was unable to see her new-born baby due to having to self-isolate which added further distress. Fortunately, the swabs were chased in a timely manner and once she was confirmed negative after five days, she was allowed to see her baby.

\section{INVESTIGATIONS}

A full septic screen was conducted which included inflammatory markers, lactate and relevant cultures. Her inflammatory markers were elevated (initial C-reactive protein (CRP) $285 \mathrm{mg} / \mathrm{L}$, white cell count $21.7 \times 10^{9} / \mathrm{L}$ ) but lactate remained normal. Electrolyte levels remained mostly within range throughout her stay. Cultures included blood cultures, urine specimen, vaginal swabs, stool culture and screening for atypical pneumonia organisms (ie urinary legionella and pneumococcal). Sputum was unable to be obtained as she did not have a cough. These all came back negative.

An abdominal X-ray was requested which demonstrated dilated bowel loops with the caecum measuring $11.4 \mathrm{~cm}$ (figure 1). Subsequently she had a computerised tomography (CT) of the abdomen and pelvis which diagnosed large bowel loop dilatation with no focal point of obstruction (figure 2). This also highlighted right sided lower lobe collapse and right sided consolidation pointing to HAP as the source of infection. A chest X-ray was requested which correlated with the CT findings. She was monitored with repeat blood sampling and repeat CTs to assess the progress of the caecal dilatation.

\section{TREATMENT}

Initially this patient was treated as per maternal sepsis guidelines with intravenous (IV) fluids, IV antibiotics and oxygen therapy for hypoxia. After surgical review, glycerine suppositories and phosphate enemas were recommended to try and promote bowel movements. Once this was unsuccessful, attempts were made to pass a flatus tube which provided minimal relief. She developed vomiting a few days into admission and a nasogastric tube (NGT) was placed which drained large amounts of bilious fluid.

As this patient was kept nil by mouth (NBM), she was reviewed by the dieticians and deemed a candidate for total parental nutrition (TPN) due to being high risk of refeeding syndrome. At this point she had been NBM for four days but no major electrolyte disturbances were noted. Prior to TPN commencing her potassium was 3.3 and her 


\section{Diagnosis and management of Ogilvie's Syndrome post caesarean section: case report}

Leah Hawkins, Sunny Ajayi

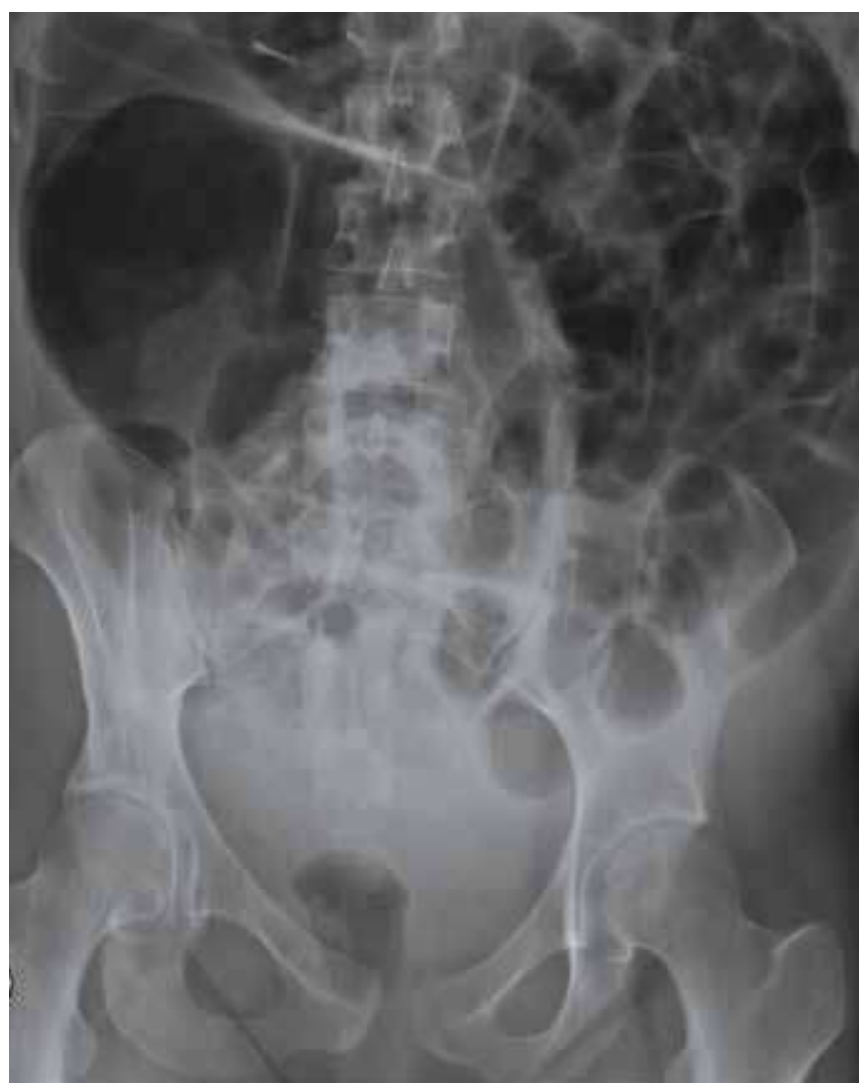

Figure 1: Initial Abdominal plain film $\mathrm{X}$-ray demonstrating $11.4 \mathrm{~cm}$ dilatation of the caecum.

phosphate was 0.77 but sodium, calcium and magnesium were all within range; she was therefore commenced on TPN and also received chest physiotherapy.

Flexible sigmoidoscopy was considered but this was abandoned as she was clinically improving and the risks of perforation were thought to be too high. She made a good recovery and no longer required TPN, NGT or flatus tube. She was discharged home after 10 days in hospital with no formal follow-up required.

\section{DISCUSSION}

OS presents similarly to bowel obstruction and can be difficult to diagnose based on signs and symptoms alone. Definitive diagnosis is made following imaging when an obvious site of obstruction cannot be identified in the presence of massive caecal dilatation. ${ }^{3}$

Management of OS includes conservative, medical or surgical intervention, based on the clinical picture figure 1. ${ }^{4}$ Literature suggests that conservative management is the mainstay of treatment in the absence of peritoneal signs. $^{2,4,5}$ This involves monitoring of vital signs, regular monitoring of CRP and electrolyte imbalances as well as consideration of NGT and flatus tube placement. Medical management involves the use of anticholinesterase inhibitors, namely neostigmine, which has shown to be effective in the treatment of gut dysmotility. ${ }^{4}$ Its use needs to be weighed up with its side effect profile as it can cause bradycardia, hypotension and vomiting. ${ }^{3,4}$ Mortality arises most notably when complications such as perforation

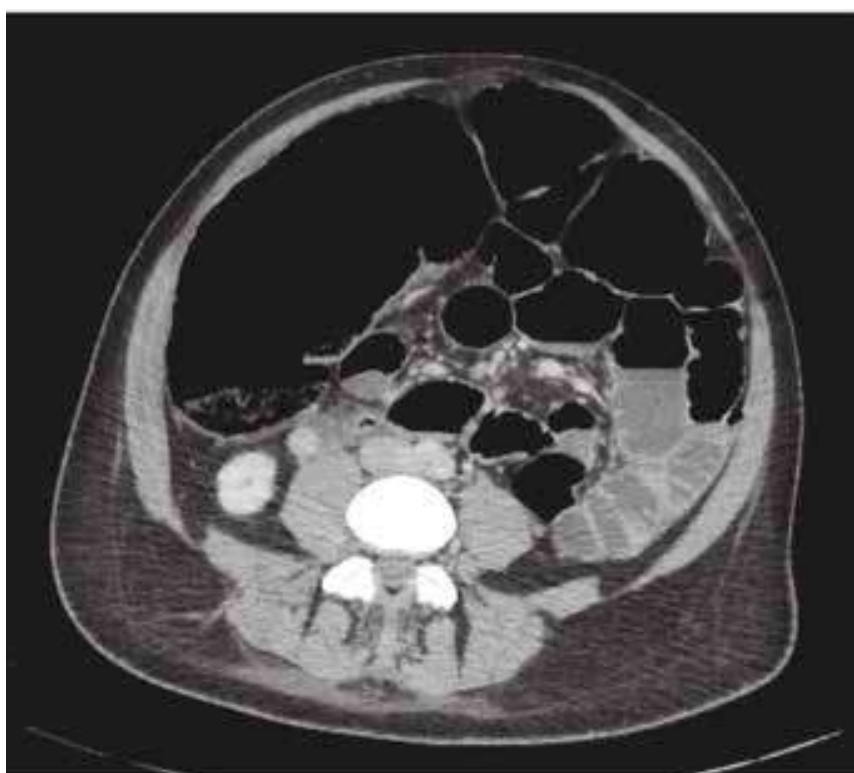

Figure 2: Computerised tomography of abdomen - showing massive dilatation of the caecum correlating with $\mathrm{X}$-ray findings.

or ischaemia occur ${ }^{2,3}$ and is also the main indication for surgery. ${ }^{1}$ Surgery can also be considered when conservative and medical management has failed., 4 Perforation secondary to OS occurs in $1-3 \%$ of patients ${ }^{4,6}$ and it has been proposed that perforation is more likely if colonic diameters are greater than $12 \mathrm{~cm} .^{2,4}$ With regards to this case, the patient improved with conservative management alone without the need for neostigmine or surgery.

Proposed risk factors leading to OS include complicated surgery, electrolyte disturbances and narcotic use. ${ }^{7} \mathrm{OS}$ is a rare complication of surgery with the incidence rate being unknown ${ }^{1,6}$ with men being more affected than women $^{1,4}$ and average age of affected patients ranging in the $50 \mathrm{~s}$ and $60 \mathrm{~s} .{ }^{4,6,7}$

The aetiology for OS is not completely understood but it is thought that there is a reduction in gut motility secondary to parasympathetic dysfunction of the gut., ${ }^{4}$ This could possibly explain why the use of neostigmine has shown improvement in these patients. There have been suggestions that OS is associated with hypokalaemia and hypomagnesaemia, but it is unclear on whether pseudoobstruction is a cause or as a consequence of this. Similar correlation has been suggested regarding raised CRP, which is commonly seen in OS. ${ }^{3}$

It was shown from the operation note that this woman had adhesions from her previous CS and the procedure was not as straightforward as anticipated. Therefore, it would have been prudent to keep this woman in for longer than other routine sections to monitor for complications. It is important to recognise early in such patients the need for surgical intervention to reduce patient morbidity and mortality.

Also, it was evident from early on that the midwives found the management of this lady rather difficult due to the nature of her clinical picture. She was later transferred to a surgical ward where the nurses were more aptly trained to manage such surgical complications and where high dependency unit input was present. 
There was a lot of confusion surrounding the use of personal protection equipment and visitation allowances due to this occurring in the early stages of the coronavirus pandemic. This in itself delayed transfer to more suitable wards and added undue stress to an already complicated case. Nevertheless, all staff involved were professional and addressed concerns appropriately to make sure this woman was managed safely and within protocol.

\section{LEARNING POINTS}

- This is a typical presentation of a patient with OS with the added complication of HAP and lower lobe collapse.

- Multiple teams were needed to ensure optimum management especially due to the multiple postoperative complications demonstrated in this patient.

- Extra precautions were needed due to protocols put in place during the coronavirus pandemic.

- Communication was essential in this case to ensure all information was conveyed to the patient and the plan surrounding her care was explained.

Correspondence to: leah.hawkins@mbht.nhs.uk sunday.ajayi@mbht.nhs.uk

\section{REFERENCES}

1. Newson, L. (2015). Ogilvie's Syndrome. Patient UK. Available at: https://patient.info/doctor/ogilvies-syndrome (accessed 11.05.2020).

2. Jayaram P, Mohan M, Lindow S, Konje J. Postpartum acute colonic pseudo-obstruction (Ogilvie's Syndrome): a systematic review of case reports and case series. Eur J Obstet Gynecol Reprod Biol. 2017;214:145-149. Available at:https://www.ncbi.nlm.nih.gov/pubmed/28531835 (accessed 11.05.2020).

3. Hughes A, Smart NJ, Daniels IR. Acute colonic pseudo-obstruction after caesarean section: a review and recommended management algorithm. The Obstetrician \& Gynaecologist, 2019;21(4):pp.283-290.

4. Maloney N, Vargas HD. Acute intestinal pseudo-obstruction (Ogilvie's syndrome). Clinics in colon and rectal surgery, 2005;18(2):96-101. Available at: https://www.ncbi.nlm. nih.gov/pmc/articles/PMC2780141/ (accessed 11.05.2020).

5. Tenofsky PL, Beamer L, Smith RS. (2000). Ogilvie syndrome as a postoperative complication. Arch Surg, 2000;135(6):682-6. Available at: https://www.ncbi.nlm.nih. gov/pubmed/10843364/ (accessed 11.05.2020).

6. Remy P. (2012). Ogilvie Syndrome. NORD (National Organization for Rare Disorders). Available at: https:// rarediseases.org/rare-diseases/ogilvie-syndrome/ (accessed 11.05.2020).

7. Haj M, Haj M, Rockey DC. (2018). Ogilvie's syndrome: management and outcomes. Medicine, 2018;97(27):e11187. Available at: https://www.ncbi.nlm.nih.gov/pmc/articles/ PMC6076157/ (accessed 11.05.2020).

\title{
Post Covid-19 Guillain-Barre Syndrome: case report
}

\author{
Dr Robert Jones, MBBS, Foundation Year 2, FGH, Dr Hannah Kolbe, MBBS, GPST2, FGH \\ Dr Alan Barton, MB Bch, Consultant General Internal Medicine \& Geriatrics, FGH
}

\begin{abstract}
This is a case report of a 62-years-old male who developed neurological symptoms consistent with Guillain-Barre Syndrome following a hospital admission for Covid-19. As a new condition we feel there are learning points to be gained for clinicians managing patients recovering from Covid-19 and discuss the symptoms, signs, and management taken in this case. The patient thankfully made a full recovery, but his case is discussed in the context of other cases in the literature. We feel that this case is different and notable as it had a longer latent period between Covid-19 resolution and Guillain-Barre Syndrome onset than any other cases reported.
\end{abstract}

\section{HISTORY}

A 62-years-old male was brought to the Emergency Department by ambulance with a 3-day history of worsening weakness and sensory deficit of the upper and lower limbs.

On Saturday he had noted weakness and paraesthesia in his hands and feet. By Sunday, the weakness extended proximally in all four limbs so that he needed his wife's help to stand. By Monday he couldn't stand even with the help of his wife, and on Tuesday his sensory and motor symptoms in the hands and feet had worsened to a point where he contacted emergency services.

He had been recently discharged 15 days earlier following a 10 day inpatient stay for chronic obstructive pulmonary disease (COPD) exacerbation where he also tested positive for Covid-19 on admission. He had no associated diarrhoea on admission but had some diarrhoea whilst an inpatient. He had been recruited into the Recovery trial ritonavir arm. He recovered well and was discharged and had been well in the community.

$\mathrm{He}$ is a known patient of COPD, hypertension (HTN), obstructive sleep apnoea (OSA), using home continuous positive airway pressure (CPAP), and is on warfarin due to previous pulmonary embolism.

\section{EXAMINATION}

His upper limb power was reduced to grade 2 bilaterally at the shoulders, grade 3 at the elbows, and grade 4 wrist and hands. Lower limb power was grade 3 at the hips, 4 elsewhere. Sensation was reduced in the c6/7 dermatomes right arm and c6/7/8 left arm. In the lower limbs sensation was reduced in a glove and stocking fashion. He displayed areflexia even with reinforcement. Peak flow was 2101/ $\min$. 